\title{
Les Eaux et Forêts sénégalais entre participation et militarisation
}

Ethnographie d'une réforme

\section{Giorgio Blundo}

\section{OpenEdition \\ Journals}

Édition électronique

URL : http://journals.openedition.org/anthropodev/481

DOI : 10.4000/anthropodev.481

ISSN : 2553-1719

Éditeur

APAD - Association pour l'anthropologie du changement social et du développement

Édition imprimée

Date de publication : 1 avril 2014

Pagination : 185-223

ISBN : 979-10-93476-00-1

ISSN : 2276-2019

Référence électronique

Giorgio Blundo, «Les Eaux et Forêts sénégalais entre participation et militarisation », Anthropologie \& développement [En ligne], 37-38-39 | 2014, mis en ligne le 01 décembre 2016, consulté le 30 avril 2019. URL : http://journals.openedition.org/anthropodev/481 ; DOI : 10.4000/anthropodev.481

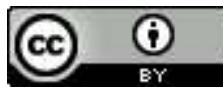

La revue Anthropologie \& développement est mise à disposition selon les termes de la Licence Creative Commons Attribution 4.0 International. 


\title{
Les Eaux et Forêts sénégalais entre participation et militarisation
}

\section{Ethnographie d'une réforme}

\author{
Giorgio Blundo
}

S'appuyant sur un long travail ethnographique, l'article aborde la réforme de "militarisation" des services forestiers au Sénégal comme une porte d'entrée pour comprendre le devenir de cette administration, son fonctionnement quotidien et sa culture professionnelle. Derrière l'apparente contradiction entre l'adoption d'un statut paramilitaire et la promotion de politiques participatives dans la gestion des ressources forestières par les bailleurs de fonds, émergent les finalités officielles et officieuses de la réforme: renforcer l'ordre et la discipline et promouvoir la "bonne gouvernance", mais surtout redorer le blason du corps, retrouver la "grande famille forestière ", se protéger des pressions politiques et rehausser les salaires des fonctionnaires. Si les réformes imposées par le haut ont partiellement échoué, l'observation de la gouvernance quotidienne des services forestiers suggère l'émergence de micro-réformes locales, qui formalisent des habitus informels et qui promettent d'être plus efficaces.

Based on a long-term ethnography, the article takes the militarization reform of forestry services in Senegal as an entry point in order to understand the becoming of this administration, its daily functioning and its professional culture. The autor suggests that the two recent reforms - militarization and decentralization of forestry resources promoted by donors- are only apparently contradictory : besides the official goals as strengthening order and discipline and promoting good governance, foresters expected that reform would restore the prestige of the service, contribute to rediscover the 'big forestry family', protect the service from political pressures, but above all, that it would increase their salaries. If top-down reforms have partially failed, the emergence of bottom-up micro-reforms can be observed occasionally. There appears to be significant scope for local innovations, which, in fact, formalize some of the more constructive features of the informal habitus, or the way things work in reality. 


\section{Introduction}

13 avril 2005, Dakar, Forum de validation du Plan d'action forestier. Dans son discours d'ouverture, le directeur du service des Eaux et Forêts salue une nouvelle étape dans l'évolution des politiques environnementales au Sénégal, allant dans le sens d'une plus forte responsabilisation des populations locales. II rappelle que la nouvelle mission des services forestiers est d'accompagner une "gestion de proximité ". Dans les ateliers, le thème des approches participatives est amplement débattu, à côté d'autres questions plus techniques. Pourtant, dans les couloirs et lors des pauses, les conversations se focalisent sur un autre sujet, qui semble tenir à cœur à la majorité des forestiers : la nouvelle réforme du corps, qui passe de la fonction publique à un statut paramilitaire. À table, les esprits s'échauffent : un ingénieur des Eaux et Forêts travaillant dans un projet allemand peste contre cette réforme, qui s'accorde mal avec la nouvelle mission de partenariat avec les collectivités locales. Un inspecteur régional lui rétorque que ceux qui ne sont pas d'accord n'ont qu'à quitter le corps, et puis qui serait contre une augmentation des salaires ? Au diable les droits électoraux qu'on va perdre après la militarisation, d'autant plus qu'on sera finalement respectés par les autres corps militaires. Le premier jour, une bonne partie des forestiers était en uniforme. Le deuxième jour, la majorité troquera la tenue pour des costumes ou des grands boubous.

Cet événement fut ma toute première rencontre avec l'univers forestier et marqua le début de mon terrain auprès des agents sénégalais des Eaux et Forêts. D'emblée, je découvrais les débats internes au service forestier, confronté à deux réformes récentes, la décentralisation et la militarisation, dont la synchronie évoquait la figure de l'oxymore en politique publique. Comment, en effet, concilier un rôle de protection des ressources environnementales s'appuyant sur la répression et les sanctions (dans le sillage de la foresterie coloniale) et un nouveau rôle " participatif ", qui transforme l'agent des Eaux et Forêts en conseiller technique des producteurs et des élus locaux ? Ces deux réformes apparemment contradictoires, l'une subie, l'autre désirée depuis longtemps, vont servir dans ces pages de point de départ et de fil conducteur à l'analyse du repositionnement actuel de l'administration forestière. 
Ce texte s'appuie sur les résultats d'une ethnographie comparative des services forestiers que j'ai menée entre 2005 et 2012 au Sénégal et au Niger. ${ }^{1}$. L'étude d'un corps de l'État, de surcroît paramilitaire, pose des problèmes particuliers que je ne peux pas aborder dans ce cadre. II faut néanmoins rappeler qu'il est impensable de prétendre mener des enquêtes sans avoir obtenu des autorisations par la Direction des Eaux et Forêts. L'expérience $\mathrm{m}^{\prime} \mathrm{a}$ prouvé d'ailleurs que même une autorisation rédigée par le plus haut responsable des services forestiers ne met pas à l'abri d'un refus courtois mais ferme de la part des subordonnés. Le traditionnel devoir de réserve, commun à tout agent de l'administration, est ici renforcé par le poids de la hiérarchie militaire. À plusieurs reprises, des agents ont tenté d'éviter mes questions, en argumentant que, puisque j'avais déjà interviewé leurs supérieurs hiérarchiques, ils ne pouvaient rien ajouter à cette parole bien plus autorisée que la leur. En effet, il a fallu, pour gagner la confiance des cadres dirigeants et avoir accès au terrain, adopter dans un premier temps la posture du "studying up " (Nader, 1974) et commencer par des entretiens au sommet de la " grande famille forestière ». Cette stratégie s'est avérée fructueuse à plusieurs égards. Elle m'a permis en particulier de reconstituer les grandes lignes de l'histoire institutionnelle et " politique " du corps et d'identifier les principaux problèmes, débats, moments de crise qui l'ont agité depuis la période des indépendances. Des missions dans les principales régions d'intervention des forestiers m'ont permis de saisir le travail quotidien des agents, jusqu'à la plus petite unité administrative, correspondant le plus souvent à un poste forestier éloigné des principaux centres urbains, doté de moyens infimes et chargé de tâches titanesques. Cette approche combinant " studying up " et "studying down " (observation non participante du travail dans les bureaux et dans les postes de contrôle, et des interactions avec les administrés) a débouché sur le "studying through " (Wedel, Shore, Feld-

\footnotetext{
${ }^{1}$ Les deux premières missions au Sénégal, en 2005 et 2006, ont été effectuées dans le cadre de l'Action thématique programmée (ATP) 2004-2006, "Étude des transferts de gestion des ressources naturelles de l'État aux collectivités locales en Afrique de l'Ouest: Niger, Mali, Sénégal ", financée par le CIRAD et conduite sous la direction scientifique de Laurence Boutinot. Les missions de recherche suivantes, élargies au Niger, se sont déroulées dans le cadre du programme «Africa Power and Politics» (APPP), un consortium de recherche financé par le UK Department for International Development (DFID) et Irish Aid. Les idées présentées ici ne reflètent pas forcément les positions du DFID, de Irish Aid ou du APPP.
} 
man \& Lathrop, 2005). Autrement dit, elle a permis de voir comment des réformes - dans le cas d'espèce la militarisation et la décentralisation de la gestion des ressources naturelles - ont été vulgarisées, comprises, négociées, mises en pièces, digérées ou partiellement escamotées par les différents niveaux administratifs des services forestiers.

\section{Des paramilitaires sans statut}

Fer de lance de la mission civilisatrice de la France en terre ouest-africaine, le service colonial des Eaux et Forêts garde le prestige et les attributs du plus ancien corps de la métropole, dont la création remonte au Moyen Age (AIGREF, 2001) : une organisation de type paramilitaire, dotée de grandes compétences techniques et scientifiques et investie d'une mission de protection et d'aménagement des forêts en vue de leur exploitation rationnelle (Boutinot, 2002).

Si la première législation coloniale sur les forêts date de juillet 1900 , octroyant aux populations locales un simple droit d'usage et de cueillette des produits sans intérêt commercial, pendant les premières trois décennies du XXe siècle, il n'existe pas de service forestier autonome en Afrique Occidentale Française, l'exploitation commerciale étant sous la coupe directe du Gouverneur général (Ribot, 2001 :2). Certes, dès 1901, le botaniste Auguste Chevalier avait pressenti l'importance de la création d'un corps de forestiers coloniaux, mais encore en 1920, Claveau, responsable du Service de l'agriculture, de l'élevage et des forêts du Sénégal, déplorait l'absence d'un cadre d'agents forestiers indigènes. En octobre 1930, l'inspecteur des Eaux et Forêts Alba, du cadre général de la Colonie, est affecté au Sénégal pour " mettre au point d'une manière quasi définitive une organisation et une réglementation d'ensemble pour toutes les colonies soumises à un climat analogue $~^{2}$. Mais, en attendant des études plus poussées, le même rapport énonce que " la base de l'action d'un service forestier au Sénégal sera la constitution d'un domaine forestier réservé, soustrait à tous droits d'usage des indigènes (...) de manière que le service forestier puisse travailler sur des terrains à lui » (pp. 58 et 60). On attend de ces forêts la

${ }^{2}$ Colonie du Sénégal, Service forestier, Rapport annuel de 1930, p. 58. 
production de revenus et un effet bénéfique sur le climat local. Le service forestier sera créé au Sénégal en 1932 (Foury, 1953 :14).

La politique forestière coloniale est définie formellement par le code forestier du 4 juillet 1935, qui s'applique aux colonies de l'Afrique Occidentale Française. Il arrête une catégorisation des forêts que l'État s'approprie, distinguant les forêts protégées des forêts classées ; il permet une répression plus sévère des infractions et octroie des pouvoirs de police plus importants aux agents forestiers, enfin il est censé favoriser les actions de reboisement "effectuées par les collectivités indigènes " ${ }^{3}$. Dans la pratique, les droits d'accès aux massifs forestiers des populations riveraines sont drastiquement réduits. Ce dispositif législatif répressif donne à l'État et à ses représentants des instruments de sanction importants (Buttoud, 1995 :41-42), faisant de la police forestière l'expression et l'outil du maintien de l'ordre colonial dans les zones rurales. Le profil du garde forestier français, réputé pour sa rigueur, face au laxisme dont on créditait les gardes-champêtres, est donc repris et sans doute exacerbé en Afrique (Buttoud, 1995 :45).

Entre 1935 et 1945, le service est composé d'ingénieurs des Eaux et Forêts et de contrôleurs et aides-contrôleurs des Eaux et Forêts, personnel français dont le nombre restera relativement stable : entre 1 et 4 inspecteurs (qui dirigent une inspection, correspondant à un ensemble de cercles) et entre 3 et 6 contrôleurs (qui président les cantonnements, au niveau administratif des cantons), pour des raisons entre autres budgétaires.

Le personnel indigène est constitué par les gardes forestiers, souvent d'anciens militaires, en contact direct avec la population et opérant au sein de brigades. C'est ce cadre local que l'administration cherchera à étoffer progressivement: les effectifs évoluent de 35 en 1935 à 100 en 1945. À partir de cette année, un nouveau corps indigène, celui des assistants forestiers ${ }^{4}$, voit le jour au Sénégal. II s'agit d'un personnel local assez bien

\footnotetext{
${ }^{3}$ Colonie du Sénégal, Service forestier, Rapport annuel de 1935, p. 8.

${ }^{4}$ Appartenant au cadre secondaire de l'AOF, suite à l'arrêté du 27 octobre 1938 portant organisation des services des Eaux, Forêts et Chasses en AOF.
} 
formé, recruté parmi les ressortissants de l'École normale de Katibougou au Mali (qui deviendra plus tard I'Institut Polytechnique Rural de Katibougou formant à la profession d'Ingénieur des Techniques Forestières) qui ont suivi un stage de deux ans à l'École forestière de l'AOF du Banco, en Côte d'Ivoire. D'un assistant forestier en 1945, on passe à 7 en 1949 puis 8 en 1950. En 1955, ils sont définitivement intégrés au cadre commun supérieur des contrôleurs (souvent en qualité d'adjoints), car on reconnaît qu'ils ont une formation professionnelle supérieure aux contrôleurs français, qui ne font que quatre mois de stage en France. Ainsi, en 1955 on compte 6 contrôleurs adjoints sénégalais pour 3 français. Les gardes forestiers sont en cette même année 170 . Ce nombre est toutefois jugé insuffisant: le Conservateur des Eaux et Forêts P. Bellouard estimait en 1955 qu'un chef de cantonnement aurait dû diriger entre 4 et 5 brigades composées de 3 à 4 gardes chacune, ce qui était loin d'être le cas (Bellouard, $1955: 20)$.

Le cadre local des gardes forestiers est composé pour la plupart d'illettrés: à titre d'exemple, les résultats du concours ouvert en 1945 pour le passage de garde auxiliaire à titulaire, donnent 11 reçus sur 60 candidats: " cela donne une idée du niveau du concours, qui était très faible et de la qualité des gardes auxiliaires qui laisse beaucoup à désirer $\aleph^{5}$. Une des raisons de cette situation est le faible salaire des gardes (indice de 130 à 300), inférieur à celui des assistants vétérinaires et des moniteurs d'agriculture (indice de 200 à 470), agents aux fonctions et compétences similaires : "il en résulte aussi un recrutement difficile, médiocre en qualité, tous les bons éléments se détournant du cadre forestier ${ }^{6}$.

Mais dès les années 50 , la situation de la foresterie s'améliore. D'une part, on peut recruter du personnel, spécialement parmi les anciens soldats de la deuxième guerre mondiale. Les moyens aussi sont plus importants, et les directions des Eaux et Forêts somment leurs subordonnés d'adopter des moyens coercitifs importants et des mesures draconiennes.

${ }^{5}$ Colonie du Sénégal, Service forestier, Rapport annuel de 1945, p. 4.

${ }^{6}$ Colonie du Sénégal, Service forestier, Rapport annuel de 1950, p. 22. 
Un dispositif commun à toute l'AOF est le classement d'au moins $20 \%$ des territoires couverts de forêts (même si ces aires ne correspondaient souvent qu'à une savane très dégradée) (Fairhead \& Leach, 2000).

En outre, si jusqu'aux années 50 , l'octroi de permis et concessions restait une prérogative de l'administration centrale - les agents forestiers assurant un simple rôle de police - la deuxième conférence forestière interafricaine tenue à Pointe-Noire en 1958 recommande que les services forestiers s'occupent aussi de la gestion des massifs forestiers non classés et qu'ils aient la tâche d'élaborer des plans forestiers régionaux.

Malgré un arsenal réglementaire conséquent, l'administration forestière coloniale apparaît peu efficace sur le terrain. Le rapport annuel de 1935 estime à $50 \%$ le nombre d'amendes non payées, car beaucoup de contrevenants étant étrangers ou "sans domicile fixe " (c'est ainsi qu'on définit les Peuls transhumants...), ils s'échappent avant de s'en acquitter ${ }^{7}$. Des préoccupations quant à l'efficacité de la police forestière sont exprimées aussi dans le rapport de 1940, qui se félicite de l'intensification de l'activité de recherche et constatation des infractions, suite à l'injonction faite aux contrôleurs et aux gardes de " ne rien laisser passer d'illégal qui tombe sous leur pouvoir $\|^{8}$. A l'orée de l'indépendance, en 1957, le service forestier compte 181 fonctionnaires, dont 14 Européens et 167 Africains.

L'indépendance entraîne la progressive nationalisation du corps et son autonomisation par rapport à la métropole mais, jusqu'en 1966, la direction sera assurée par un Français. Les gardes forestiers seront progressivement remplacés, à partir de 1963, par les Agents Techniques des Eaux et Forêts (ATEF), formés à l'École des Agents Techniques des Eaux et Forêts de Djibélor (Ziguinchor). Si la création du centre de Djibélor a permis de relever les compétences techniques des agents de base ${ }^{9}$, jusqu'au début des années 70 le service manque cruellement d'effectifs dans les corps des

\footnotetext{
${ }^{7}$ Colonie du Sénégal, Service forestier, Rapport annuel de 1935, p. 5-6.

${ }^{8}$ Colonie du Sénégal, Service forestier, Rapport annuel de 1940, p. 11.

${ }^{9}$ Entre 1963 et 1992, le Centre a formé 432 agents techniques des Eaux et Forêts, dont 22 étrangers et 410 sénégalais, tous intégrés dans la fonction publique.
} 
ingénieurs : au Sénégal, on compte en 1974 quatre IEF et une vingtaine d'ITEF pour environ 300 personnels.

"Initialement, les ingénieurs des eaux et forêts sortaient de Nancy, et les ingénieurs des travaux sortaient de l'École des Barres (École Nationale des Ingénieurs des Travaux des Eaux et Forêts à Nogentsur-Vernisson) et de Bambey. Et il n'y avait pratiquement pas de passage d'ingénieur des travaux vers l'ingénieur des Eaux et Forêts. Et le système des ingénieurs des Eaux et Forêts n'était pas très attractif. Parce que pour faire Nancy, il faut faire un diplôme d'agronomie et une fois que tu as ton diplôme d'agronomie, il faut vraiment aimer la forêt pour aller faire encore deux ans et revenir et avoir le même indice. C'est-à-dire que tu fais une école d'agronomie, tu as ton diplôme d'agronome, tu es à l'indice A1. Tu fais encore deux ans là-dessus pour aller faire la foresterie, tu reviens, tu es également à A1. Donc je pense que ceci a beaucoup contribué à ne pas attirer les gens pour le faire. Mais la pression, la demande interne, pour des jeunes qui estimaient qu'ils avaient encore le potentiel pour aller étudier, comme moi, était relativement forte. Et puis, le Canada venait de s'ouvrir, par le CRDI, à la foresterie. Moi je suis allé à l'université de Laval. " (ancien directeur des Eaux et Forêts du Sénégal)

Une première hémorragie des effectifs aura lieu à partir de 1969, lorsque le président Senghor crée la Direction des Parcs Nationaux, directement rattachée à la présidence, et la confie à André-Roger Dupuy, un ancien militaire français, qui occupera le poste de conservateur en chef jusqu'en 1986. Dupuy sera le partisan d'une approche militarisée dans la lutte contre le braconnage et la conservation de la nature (Ségalini, 2012). C'est sous sa direction qu'en 1979 (loi no 79-33 du 24 janvier 1979) le personnel des Parcs Nationaux reçoit un statut militarisé. C'est un motif de frustration pour les fonctionnaires du service des Eaux et Forêts, qui acceptent difficilement de rester des "civils habillés ", titre moqueur dont ils sont affublés par les autres "corps habillés " ${ }^{10}$, alors que des agents issus des

\footnotetext{
${ }^{10}$ Répandue dans la plupart des pays d'Afrique de l'Ouest, « l'expression renvoie (...) de façon très large aux hommes en uniforme au service de l'ordre d'Etat, des soldats aux douaniers en passant par les policiers, gendarmes, forestiers, etc. » (Glasman 2011 :19).
} 
mêmes écoles de formation bénéficient des avantages de la carrière militaire.

Une première demande de réforme du statut échoue dans les années 1980, après le rejet du ministère des Finances en raison d'un coût excessif pour l'Etat. Le processus est relancé en 2002, lors d'une réunion des représentants des trois corps forestiers (agents techniques, ingénieurs des travaux et ingénieurs) organisée à Thiès sous l'égide de la direction générale. Une commission est créée, chargée d'élaborer le projet de loi et le projet de décret d'application, mais au bout d'une année de travail, les travaux s'arrêtent : la première mouture du projet de loi reste dans les tiroirs du secrétaire général du gouvernement et les ministères compétents pour l'examiner ne réagissent pas. En 2004, la grogne monte parmi les agents forestiers, lorsqu'ils se voient refuser l'indemnité de logement que l'Etat a décidé d'accorder à tous les corps paramilitaires. Une nouvelle commission, formée par deux ingénieurs et deux ingénieurs des travaux, reprend le travail en février 2005. Après des décennies d'atermoiements, le processus s'accélère prodigieusement: en six mois, le projet passe entre les mains des représentants des ministères de l'Environnement, des Finances, de la Culture et des Forces Armées, est examiné par le Conseil d'Etat, adopté sans modifications par le Conseil des ministres et voté en assemblée le 15 juillet 2005.

La loi 2005-10 du 03 août 2005 portant statut particulier du personnel des Eaux, Forêts et Chasses sanctionne ainsi la militarisation tant attendue par les agents du service forestier. Faut-il pour autant en conclure que cette réforme traduit la légitimation d'une culture professionnelle militaire et autoritaire héritée du passé colonial (Ségalini, 2012 :118-119) ? Un détour par le vécu des forestiers durant les premières années de mise en œuvre de la militarisation permettra de fournir, je l'espère, une réponse plus nuancée. Selon la place occupée dans la hiérarchie forestière, le type de trajectoire professionnelle ou l'expérience de terrain, les perceptions, les attentes et les contenus assignés à la réforme varient, et donnent lieu à des débats sur l'identité ou l'éthique professionnelle du service, sur sa réorganisation, sur ses relations avec les autres corps militarisés ou avec le public. Ces débats ont souvent tourné autour de la question du port de l'uniforme, marqueur fort de l'identité professionnelle de ces « bureau- 
crates en uniforme " (Blundo \& Glasman, 2013). La confrontation des discours sur l'uniforme et du "langage de l'uniforme " (Blundo \& Glasman, 2013), hautement polysémiques, avec l'observation des pratiques quotidiennes de l'administration forestière, permettra de dévoiler les différentes facettes de la mise en œuvre d'une réforme de militarisation intervenue parallèlement aux politiques participatives promues par les bailleurs de fonds.

\section{Retrouver l'esprit de corps, porter l'uniforme dignement}

Le premier des motifs ayant présidé officiellement à la promulgation de la loi 2005-10 du 03 août 2005 était « la nécessité de valoriser le cadre qui s'est déprécié au fil des ans en raison des départs importants des agents des Eaux, Forêts et Chasses vers d'autres cadres, notamment celui des Parcs nationaux ". Le législateur omet de mentionner que si, dès la moitié des années 70, qui marquent le début de "l'ère des projets " pour les Eaux et Forêts, l'appui des bailleurs de fonds permet à la direction de mettre en œuvre une politique de recrutement soutenue $(74,4 \%$ des forestiers en service en 2005 avaient intégré le corps entre 1970 et 1990), les politiques d'ajustement structurel mises en œuvre sous le mandat d'Abdou Diouf ont conduit à la fin des recrutements quasi-automatiques des diplômés à partir de 1992. Pendant près de 20 ans, l'Etat n'a pas pu remplacer les agents ayant quitté le service, les départs à la retraite ou les décès. Les forestiers sénégalais, qui étaient 524 en 1993, ne sont plus que 452 en 2005, répartis entre 50 Ingénieurs des Eaux et Forêts (IEF), 83 Ingénieurs des Travaux des Eaux et Forêts (ITEF), 293 Agents Techniques des Eaux et Forêts (ATEF) et 26 agents horticoles et des Parcs Nationaux. S'ajoutent à ces effectifs des agents en détachement ou en disponibilité (essentiellement dans des programmes et des projets de développement), pour un total de 77 agents supplémentaires, dont 41 appartiennent au grade des IEF ${ }^{11}$.

\footnotetext{
${ }^{11}$ Une timide politique de recrutement est en cours depuis 2003, notamment au niveau des agents de terrain (ITEF et ATEF), en raison de 15 diplômés environ par an, dont certains sont embauchés sur des contrats temporaires dans le cadre du Programme d'Appui au Développement Forestier (PADF, financé par les Pays-Bas). Les recrutements ont recommencé récemment, grâce au programme présidentiel « 15000 emplois » pour la fonction publique : ce
} 
Aujourd'hui, les services forestiers sénégalais sont un corps administratif vieillissant et qui peine à se renouveler: en 2009, plus de cinquante agents sont partis à la retraite. Les données statistiques dont nous disposons montrent qu'en $2005,61 \%$ des agents en service avaient plus de 50 ans, pourcentage qui grimpait à $88,6 \%$ pour le corps des IEF.

Mais la crise démographique du service, élément somme toute récurrent depuis le temps colonial, comme on l'a vu, ne représente qu'une dimension, sans doute mineure, de cette dépréciation que la réforme était censée corriger. Car ce qui comptait aux yeux des forestiers, notamment pour les ITEF et des ATEF, habitués à l'uniforme, c'était la reconnaissance institutionnelle des tâches et des attributions propres à une administration paramilitaire, sans laquelle ils ne resteraient, face aux douaniers, aux policiers et aux gendarmes, que des simples " civils habillés » :

"Nous mettons la tenue mais en fait nous ne sommes absolument rien, on n'est pas militarisés. (...) Pendant très longtemps on a couru derrière cette militarisation, comme nos collègues gendarmes, policiers, douaniers, service d'hygiène. Même le service d'hygiène qui n'intervient pas en brousse, il est militarisé depuis très longtemps, alors qu'ils n'opèrent que dans les maisons. Et le forestier qui est tout le temps en brousse, contre les braconniers, il affronte toutes sortes de difficultés en brousse, avec son arme et tout, bien habillé en tenue, il est civil en fait. (...) Alors c'est frustrant. C'est honteux ! Moi, j'avais même honte de mettre la tenue quand j'étais à côté de mes copains gendarmes, à côté de mes amis policiers. (...) On a des galons, mais ces galons ce n'étaient que des chiffons. Ces galons, avant, ce n'étaient que des chiffons. Parce que vous mettez des galons mais vous êtes civil » (adjudant-chef, adjoint au chef de poste de G.).

En premier lieu, l'octroi d'un statut militaire représente donc pour les agents une mise en conformité avec les réalités du service, le redressement d'une injustice, ainsi que l'entrée de plein droit dans la grande fa-

n'est qu'en 2007 que l'on a pu intégrer au service 3 IEF, 3 ITEF et 30 ATEF. La fin des projets ayant eu comme conséquence le tarissement des bourses d'études, on a désormais des difficultés à recruter des IEF. 
mille des corps paramilitaires. Le langage exprimé dans ces discours est un langage de l'homogénéité (Blundo \& Glasman, 2013 :5).

En deuxième lieu, dans le ressenti de plusieurs forestiers, la réforme de militarisation aurait permis de revitaliser un esprit de corps réputé fragilisé. En effet, en dépit des discours officiels qui exaltent l'unité de la "grande famille forestière", les rituels collectifs qui ponctuaient cette solidarité prétendue et réaffirmaient les valeurs et les missions communes du corps ont quasiment disparu. Il en est ainsi de l'initiative éphémère des "nuits vertes ${ }^{12}$, des réunions thématiques annuelles s'achevant par une soirée dansante, organisées à tour de rôle par les Inspections régionales des Eaux et Forêts (IREF). Elles étaient une occasion de rencontre, d'échange mais aussi d'expression de besoins au niveau des services déconcentrés :

"Dans le temps (...), chaque année, on se réunissait pendant deux jours, on choisit une région et l'on demande à tout le monde de se cotiser, on prend un orchestre et nous faisons une fête. Avant la fête, pendant une journée, nous faisons un audit de l'administration, c'est-à-dire que tout le monde est là, chacun dit ce qu'il pense des Eaux et Forêts, comment on peut les améliorer, quels sont les abus... etc. Mais au fur et à mesure que les gens ont pris de l'âge, ça s'est amenuisé. (...) On est à la recherche de ce passé où c'était une très grande famille. Ça j'avoue, j'ai assisté au début et à la fin de cette affaire, c'était une très grande famille, très solidaire " (IEF, Dakar, 15/4/05).

Au-delà d'un unanimisme de façade, que le devoir de réserve ne fait qu'accentuer, les services forestiers, à l'instar de tout corps social, apparaissent traversés par des conflits internes, des ressentiments et des frustrations diffus. Deux facteurs semblent y avoir contribué de manière déterminante : l'émergence d'enclaves bureaucratiques fonctionnelles créées par la coopération au développement, et une gestion des ressources humaines et des carrières perçue par les agents comme opaque, arbitraire et imprévisible.

${ }^{12}$ La première a eu lieu à Thiès en 1976 et la dernière en 1981 à Saint-Louis. 


\section{Service traditionnel et projets : une administration à deux vitesses ${ }^{13}$}

Traditionnellement, à l'instar des autres corps de contrôle militarisés (douanes, police, gendarmerie), les postes les plus brigués par les agents forestiers, définis dans le jargon local comme "juteux", étaient les " postes à forte densité de transactions, ceux où l'on est en contact direct avec les usagers, les postes de terrain plutôt que les postes administratifs » (Blundo et Olivier de Sardan, 2007 : 98). Le premier critère déterminant I'intérêt d'un poste était sa capacité à engendrer simultanément trois types de ressources financières (Cantens, 2009) : de l'argent public (les recettes domaniales, destinées à l'État et dont une partie est reversée dans un fonds forestier, et les recettes du contentieux, dont $70 \%$ est de nos jours reversé aux collectivités locales); de l'argent privé, aussi bien légal (correspondant à $30 \%$ des amendes qui est attribué aux agents indicateurs et verbalisateurs) qu'illégal (correspondant aux produit des arrangements informels et aux pots-de-vin reçus); de l'argent collectif (collecté dans chaque service dans des " cagnottes", ou caisses noires), redistribué selon des critères divers. Au Sénégal, tout forestier sait que les sites où ces trois ressources sont le plus aisément mobilisables se trouvent dans les régions de Kolda et Tambacounda, les seules toujours ouvertes à la production de charbon, ou correspondent aux postes de contrôle situés au carrefour des axes routiers les plus fréquentés (Kaffrine, Gouloumbou, Bargny, etc.).

Mais à partir de la moitié des années 70 , s'offre aux forestiers une nouvelle catégorie de postes " juteux ", qui associe au critère de l'intérêt économique celui de la qualité des conditions de travail et des opportunités de promotion: les projets de développement financés par la coopération internationale. Depuis les tout premiers programmes en Casamance (Programme forestier de Ziguinchor, 1973) et dans le Ferlo (Programme de restauration des espèces sylvo-pastorales et d'amélioration du gommier à Mbidi, 1974), jusqu'au récent Projet de gestion et de restauration des terres dégradées du bassin arachidier (PROGERT, initié en 2007), les ser-

\footnotetext{
${ }^{13}$ Pour de plus amples approfondissements sur les effets de l'aide dans la réorganisation du service forestier, voir Blundo, 2011a.
} 
vices forestiers ont été associés à, ou ont dirigé, près de 45 projets d'envergure régionale ou nationale. Certes, leurs modes d'intervention ont évolué au gré de l'évolution des approches développementistes; certes, leur bilan est souvent plus que mitigé, et il ne reste parfois de ces dispositifs d'intervention que des bâtiments défraîchis, des pancartes délavées et des cimetières de véhicules. Mais ce qu'il importe de souligner ici c'est que l'émergence de ces " enclaves bureaucratiques fonctionnelles " branchées sur le système de l'aide va constituer une source importante de différentiation au sein du corps forestier.

En dehors des avantages salariaux évidents, travailler dans un projet donne l'accès à des moyens de travail conséquents, à une plus grande autonomie, à des stages de formation continue, à des voyages d'étude et des bourses pour la formation supérieure. C'est une expérience qui étoffe le CV du fonctionnaire, et lui ouvre la porte au marché des consultations grâce aux contacts tissés. Mais c'est aussi la voie royale à la constitution ou à l'élargissement d'un réseau de clientèle, par les redistributions possibles des ressources dont disposent les projets. C'est enfin un facteur d'accélération des carrières : tous les directeurs, anciens et actuels, des Eaux et Forêts du Sénégal, sont passés par des projets et y ont conduit la plupart de leur vie professionnelle.

Le contrôle des ressources gérées par les projets devient vite un enjeu de pouvoir, à tel point qu'en 1985, le ministre de la Protection de la nature, Cheikh Abdoul Khadre Cissokho, décide de scinder en deux la direction des Eaux et Forêts pour créer une nouvelle direction, censée s'occuper de "reboisement et conservation des sols", mais qui centralise de facto tous les projets et est dirigée par un homme de confiance. Cette expérience ne dure que cinq ans : en 1990, suivant un mouvement général de regroupement, dans tous les ministères, des directions qui avaient des missions proches, la "direction des projets " est supprimée. Un nouveau ministre, Moctar Kébé, procède à la fusion.

En dépit de cette réforme, ce système a donné lieu à des conflits internes. Ces conflits éclatent à plusieurs niveaux. 
Ils peuvent avoir pour objet l'affectation à des postes de responsabilité dans les projets, qui peut suivre, tout au moins dans les imaginations, les pentes du clientélisme et du favoritisme. Les projets n'ont pas échappé à la règle officieuse selon laquelle la nomination à des postes " intéressants " engendre des obligations en retour ou même des formes de versement d'une rente régulière vers le haut de la hiérarchie.

Ils opposent aussi le Ministre de tutelle et le directeur national, quant à la récupération d'une partie des ressources des projets : selon un exdirecteur des Eaux et Forêts, " un ministre qui voit un projet, ce qu'il veut surtout, c'est des véhicules et de l'essence pour aller faire sa politique ". Outre le carburant et des aides mineures, la passation des marchés publics permet des arrangements avec des fournisseurs complaisants, des opérations de surfacturation et le financement de la politique.

Des frictions interviennent également entre projets et services forestiers déconcentrés: jusqu'à la fin des années 1990, un projet de reboisement intervenant au niveau d'un arrondissement sénégalais pouvait avoir des moyens logistiques plus importants qu'une inspection régionale. Les services traditionnels quémandaient des aides aux projets, alors qu'ils étaient censés les encadrer et les superviser. À partir du moment où les projets appuyaient l'administration, ils étaient les instruments de sa mise en dépendance. Aux frustrations des ingénieurs de conception s'ajoutaient celles des agents d'exécution : à Kolda, certains ATEF refusaient de collaborer avec les animateurs du PROGEDE (Programme de gestion durable et participative des énergies traditionnelles et de substitution), mieux payés et dotés de meilleurs moyens, ou alors réclamaient de l'essence et d'autres faveurs pour se déplacer.

L'administration forestière fonctionnant à deux vitesses, l'écart s'est creusé entre le service "traditionnel ", délabré, sans moyens, relégué à faire de la police forestière, et les services des " projets ", qui devenaient de plus en plus convoités. Dans ce contexte, la démotivation a gagné les agents restés en dehors du système des projets, encourageant parfois la recherche de formes de rétribution parallèles ou illicites, et facilitant le développement d'espaces de soupçon au sein du corps. 
Mais le cycle des projets est désormais terminé (il ne reste plus que quatre programmes forestiers au Sénégal ${ }^{14}$ ) et depuis 2003 les services forestiers sénégalais sont entrés dans la période de l'appui budgétaire en partenariat avec la coopération hollandaise. Si cette nouvelle phase a permis un meilleur équipement logistique des inspections régionales et de la direction nationale, en même temps la plupart des activités de renforcement institutionnel (séminaires, formations, réunions de sensibilisation, appui aux producteurs) ont été arrêtées, à cause de la suppression du système des per diem et du retour aux barèmes de l'administration.

Dans un contexte de réduction des moyens de l'administration forestière, de raréfaction des conditions de travail valorisantes et de perte des avantages tirés de la rente du développement, la militarisation a représenté, pour les cadres dirigeants, un moyen de motiver financièrement les agents, dont le revenu est quasiment doublé par les primes et indemnités inhérentes au statut paramilitaire.

\section{Mettre de l'ordre dans la gestion des ressources humaines ${ }^{15}$}

Dans un service de contrôle et de répression, les agents sont appelés à changer fréquemment de poste de travail, officiellement pour éviter de développer des accointances avec les populations. La pénurie du personnel et de postes convoités fait des affectations un véritable casse-tête. Lorsqu'on travaille sur les biographies professionnelles des agents forestiers ${ }^{16}$, on est surpris par la richesse des parcours, tant sur le plan des postes recouvrés que des localités où l'on a servi. Mais ce qui frappe est la grande variabilité dans la durée occupée dans les différents postes, ainsi que les parcours en dents-de-scie (postes de haute responsabilité suivis de postes

\footnotetext{
${ }^{14}$ PROGERT (projet de gestion et de restauration des terres, PNUD, dans le bassin arachidier) ; PROGEDE, en attente de la deuxième phase ; programme Wula Nafaa, de I'USAID ; projet de développement fruitier, projet national à Samba Sarr.

${ }^{15}$ Sur la gestion des ressources humaines dans les services forestiers ouest-africains, voir Blundo, 2011b.

${ }^{16}$ Nous avons réalisé, au cours de nos recherches, 150 biographies professionnelles sur les carrières des agents, et disposons pour le Sénégal d'une base de données informatisée concernant les carrières de 380 agents.
} 
de grade inférieur). Cela est présenté par les forestiers comme une exigence de souplesse et de capacité d'adaptation, et n'est pas discuté puisqu'on invoque la discipline militaire.

Les mouvements de personnels sont très fréquents. Le ministre décide de l'affectation des ingénieurs et des ingénieurs de travaux, tandis que le directeur général des Eaux et Forêts affecte les agents techniques, sous la proposition des inspecteurs régionaux. Dans la décennie 95-05, il y a eu 161 mouvements nationaux au Sénégal. En moyenne, il y a eu 68 mutations par an, mais à l'occasion des remaniements ministériels ou des changements dans la direction, ce chiffre peut concerner plus de 100 agents, c'est-à-dire un cinquième des effectifs.

Chaque nouveau directeur s'entoure de nouveaux collaborateurs, selon des logiques clientélistes classiques (promotionnaires, hommes de confiance, originaires de la même région, militance politique). Ces affectations à base clientéliste peuvent engendrer de la confusion dans les hiérarchies et nuire à l'efficacité du service. Mais une autre conséquence du clientélisme est qu'il empêche les sanctions directes. De ce fait, des mouvements d'affectation peuvent dissimuler des sanctions, ce qui a pour conséquence, comme dans un jeu de domino, de déplacer sur la carte du pays d'autres agents au comportement irréprochable. Par ailleurs, les sanctions ne frappent pas seulement les agents "véreux " ou "indélicats ". Si ces derniers savent redistribuer les fruits de leurs activités illicites, ils peuvent garder leur poste pendant des années. Mais on peut également relever de leurs fonctions des agents réputés trop rigides, peu enclins aux compromis, et qui se sont trop souvent opposés aux injonctions du directeur lorsqu'ils estimaient qu'elles contrevenaient à la déontologie professionnelle.

Le clientélisme n'est toutefois pas le seul critère qui dicte les affectations. D'une part, un nombre considérable d'agents reste exclu des réseaux de clientèle, et accepte militairement d'aller là où les besoins du service l'appellent, même si les raisons des mutations successives leur échappent et démotivent à la longue les fonctionnaires. D'autre part, les nominations à des postes se font aussi en fonction des compétences des agents et de leur expérience : il est courant qu'on envoie dans une zone réputée difficile 
un agent expérimenté pour régler un conflit en cours ou réparer les dégâts commis par son prédécesseur. Il semble, par exemple, qu'en 2000, la direction aurait envoyé dans les inspections situées en zone forestière, qui connaissaient beaucoup de problèmes, des agents formés essentiellement dans les projets, pour redresser leur gestion.

La direction essaye donc de jongler avec des impératifs apparemment contradictoires : satisfaire les pressions des clientèles et assurer un fonctionnement acceptable du service. En règle générale, les postes au turnover le plus élevé se trouvent dans les zones de l'exploitation forestière. Les agents sont vite mutés lorsqu'il s'avère qu'ils ont trempé dans les combines, s'ils se sont opposés à un puissant exploitant forestier, mais aussi si le poste est convoité par un autre agent ayant "le bras plus long ». En revanche, certains forestiers restent longtemps en service dans des postes clef, en particulier les gérants des caisses intermédiaires. Ils assurent ainsi la stabilité et la mémoire administrative du service, dans un environnement où les archives administratives sont mal tenues ou inexistantes.

Enfin, les affectations peuvent aussi tenir compte de demandes précises de la part des agents, mais ces cas seraient officiellement assez rares, et se limiteraient à des raisons qu'on appelle pudiquement "sociales" : maladie, proximité de la retraite, parents âgés ou malades, éloignement excessif de la famille, etc. En réalité, ces cas sont plus fréquents qu'on veut bien l'admettre : selon l'adjoint au directeur des Eaux et Forêts du Sénégal, lors d'un mouvement national récent concernant 96 ATEF, 30 avaient demandé à être mutés, et l'on a satisfait leur requête. En respect de ces mêmes critères " sociaux ", les épouses des agents, si elles sont aussi fonctionnaires, sont systématiquement affectées dans la même localité, même si elles sont en surnombre.

Anomique, arbitraire, implacable voire inhumaine : $c$ 'est ainsi que les agents forestiers caractérisent la gestion des hommes par les cadres dirigeants. On comprend mieux pourquoi la militarisation a été investie par la base d'attentes et de revendications, sans doute démesurées, axées sur une « mise en ordre » de la gouvernance interne du service. D'une part, la réforme a permis, de façon plutôt concluante, de continuer et achever le processus de reconstitution des carrières administratives initié par le dé- 
cret $n^{\circ}$ 2003-180 du 15 avril 2003. Ce décret classe désormais les Ingénieurs des Eaux et Forêts en trois corps distincts (hiérarchie A1, A2 et A3), permettant ainsi de reconnaître des diplômes obtenus à l'étranger dans de nouvelles filières de formation à l'international (Canada, Etats-Unis, mais aussi Cameroun, Nigeria et pays du Maghreb). D'autre part, on attendait d'elle qu'elle réinstaure l'ordre dans la hiérarchie militaire :

"Les gens portent la tenue et les galons n'importe comment! Le galon coûte juste 3000 FCFA, il est vendu par des gens du service et comme c'est le même prix, pourquoi se priver de mettre un galon supérieur? " (chef de secteur)

"Les tenues ne sont plus respectées! Quand un responsable de projet s'attribue de galons même s'il est inférieur à l'inspecteur, puisqu'il est en prise directe avec le ministre, il y a relâchement et le respect manque » (ancien directeur des Eaux et Forêts)

Ces lamentations font écho à celles sur les uniformes débraillés ou dépareillés, conséquence des dotations annuelles qui parviennent au compte-goutte, problème récurrent que la militarisation n'a pu enrayer. Mais il serait trompeur de n'y voir que les signes d'une culture militaire revitalisée. Derrière l'allusion aux insignes et grades militaires, se niche l'espoir que "peut-être il y aura encore du sérieux dans ce que nous faisons, dans le travail, le respect de la déontologie, tout ça, fournir plus d'effort encore... » (chef de brigade).

\section{"Geler les convoitises sur les domaines et sur les compétences des forestiers »}

Administration de répression d'inspiration coloniale, le corps des Eaux et Forêts a intégré progressivement des tâches propres à une administration de développement et a épousé, au fil du temps, ses différentes approches : de projets en régie technocratiques où la seule forme de participation était le food for work, on est passé à des projets de sensibilisation ou d'animation, où l'on expérimente la MARP (méthode active de recherche participative), jusqu'à la phase actuelle de décentralisation. 
À la suite des nouvelles politiques de décentralisation, qui placent les communes rurales au centre de la gestion des ressources naturelles, le forestier est désormais appelé à devenir un animateur et un accompagnateur des populations rurales dans leurs choix relatifs à l'exploitation de leur environnement. Depuis 1996, les collectivités locales, ou pour le moins celles situées dans les régions de Tambacounda et de Kolda, sont censées gérer près de $55 \%$ de l'espace forestier sénégalais, représenté par les forêts communautaires (Boutinot et Diouf, 2007). Cette prérogative est toutefois conditionnée par la réalisation d'un plan d'aménagement ${ }^{17}$. En outre, les présidents des communautés rurales statuent sur toute demande d'abattage d'arbres situés dans le domaine forestier communautaire. Enfin, les collectivités locales bénéficient des sept dixièmes des produits issus des infractions perpétrées dans les forêts sous leur juridiction. Désormais, les communautés rurales et les groupements villageois peuvent investir une filière traditionnellement contrôlée par un oligopole d'exploitants forestiers regroupés en une puissante fédération.

Plusieurs études récentes ont cependant montré que la décentralisation de la gestion des ressources forestières rencontre de sérieux problèmes et est loin d'être effective (Kante, 2006, 2009, 92008, 2007, Boutinot, 2002, 2005, Boutinot et Diouf, 2006). On connaît moins, en revanche, la position des forestiers sur la question. En la matière, le double langage est une constante. Tout forestier que j'ai rencontré a, dans un premier temps, énoncé la vulgate officielle, qui exalte la décentralisation et la participation locale. Mais une fois que les langues se délient, le tableau qui se dessine montre une administration forestière méfiante vis-à-vis du rôle exercé par les collectivités locales, accusées de manquer de sensibilité pour les questions environnementales, de monnayer les autorisations d'exploitation, de ne pas prévoir dans leurs budgets l'appui aux brigades, de ne pas inviter les forestiers à participer aux réunions de délibération des conseils ruraux, et d'abuser de leurs nouvelles prérogatives à des fins politiciennes ou d'enrichissement personnel. En dépit d'un discours de plus en plus " participatif ", le sentiment partagé par les agents des Eaux et Forêts

\footnotetext{
${ }^{17}$ Pour l'heure, on dispose de sept plans d'aménagement seulement, qui couvrent 300000 ha
} de forêts, soit 20\% des forêts communautaires (Boutinot, 2005 et Boutinot et Diouf, 2007). 
oscille entre le regret pour une décentralisation inachevée et la conviction que "l'on ne transfère pas l'environnement ", ce domaine devant rester une compétence réservée à l'État.

En même temps, même si la majorité des personnels, tous niveaux confondus, était favorable à la réforme de militarisation, le terrain a dévoilé des tensions internes et l'existence de groupes dissidents qui considéraient la réforme comme un retour vers un passé révolu, en contradiction avec le nouveau rôle "participatif » conféré aux Eaux et Forêts. Cette minorité était composée des ingénieurs ayant fait l'essentiel de leur carrière dans les projets et /ou étant rentrés dans le corps par concours direct, après la formation universitaire. D'une part, ces cadres dissidents craignaient que le renforcement de la dimension paramilitaire ne porte préjudice à leur activité de consultants auprès des grandes institutions internationales consacrées à la protection de l'environnement. D'autre part, ils considéraient que le port désormais obligatoire de l'uniforme en toutes circonstances, même dans des activités de conseil et encadrement des populations, risquait de brouiller les relations avec leurs interlocuteurs villageois. Le langage sur l'uniforme faisait référence ainsi à la question de la " juste distance » (Blundo \& Glasman, $2013: 5$ ) entre les fonctionnaires et leur public :

« De 1978 à 2002, j'étais dans les projets. Cela a induit une petite déformation sur moi. Donc la tenue n'est pas mon fort. Quand je l'ai mise, la population ne me reconnaissait pas. Alors que je rentrais dans l'intimité des gens, à travers les activités dans les projets. Je me rappelle qu'une fois, dans la zone sylvo-pastorale, nous avons eu une panne. Au lieu de nos aider, les gens ont fui, car nous étions en tenue. Si on nous avait donné les mêmes avantages financiers, mais sans la militarisation, je pense que certains auraient accepté " (ITEF, adjoint à I'IREF de D., 30/7/09).

La tenue est un symbole de l'autorité et de ce monopole que l'État revendique sur la gestion exclusive des ressources naturelles. Appliquer des sanctions, arrêter des contrevenants, se défendre contre des attaques physiques, autant de contextes dans lesquels l'uniforme est considéré comme indispensable. Mais les forestiers sont porteurs d'une identité fluctuante : de par leur histoire, la diversité de leurs missions et la richesse 
des trajectoires individuelles (alternance entre postes de terrain et bureaux, entre police forestière et projets), ils sont partagés entre l'identité militaire et l'identité civile. Ainsi, les deux identités sont mobilisées selon les enjeux : on est militaire lorsqu'il s'agit de se retrancher derrière le devoir de réserve face au chercheur ou au journaliste, on est militaire lorsqu'on doit sermonner ou sanctionner un paysan, mais on est civil pour répondre à un appel d'offre d'une consultation, dialoguer avec les bailleurs de fonds, s'intégrer dans le tissu social d'une localité ou justifier la clémence manifestée à l'égard d'un proche ou d'un patron.

Confronté à la redéfinition de ses compétences - interprétée comme une perte d'autorité et de ressources économiques - le corps des Eaux et Forêts se sent menacé. Certains agents soupçonnent même des groupes de pressions d'œuvrer pour le déclassement de toutes les forêts, et craignent, à terme, la disparition pure et simple du service. De ce fait, la militarisation a représenté pour beaucoup un moyen de "maintenir le corps en place. $C^{\prime}$ est une soupape de sécurité pour geler les convoitises sur notre domaine et sur nos compétences " (AN 22/9/06).

En effet, les pouvoirs discrétionnaires conférés aux agents dans l'application du code forestier sont fortement influencés par des interventions venant d'une pluralité d'institutions et d'acteurs, formels et informels. Ces interventions peuvent avoir comme auteur des autorités religieuses ou coutumières, des autorités du commandement territorial, comme les préfets et les gouverneurs, des députés, des hauts gradés de l'armée, ou encore des collègues forestiers. Elles ont souvent l'effet d'annuler ou de transformer une décision administrative ou une sanction effectuées dans le respect de la loi. J'ai montré ailleurs (Blundo, 2012) comment l'influence de ces logiques de redevabilité multiples et parfois contradictoires débouche sur une action publique caractérisée par l'hybridation des normes et l'informalisation des procédures administratives, tout en démotivant des agents qui se voient emprisonnés dans un faisceau d'injonctions contradictoires (Bierschenk, 2010) : faire appliquer le code forestier tout en devant constamment déroger à ce même code lorsqu'il dérange les intérêts des citoyens en mesure de solliciter l'intercession d'une autorité influente. 
Dans un contexte qui a vu, dans la dernière décennie, une forte politisation du corps forestier, et un accroissement des interventions extérieures dans le fonctionnement du service au quotidien, "la tenue est aujourd'hui une protection pour les agents " (cadre de la direction générale des Eaux et Forêts), une manière aussi de réaffirmer leur centralité face à d'autres acteurs revendiquant leur part dans la production de l'étaticité. On comprend ainsi la réticence du service forestier face aux demandes des éco-gardes, chargés de faire respecter les conventions locales dans les zones mises en défens dans les territoires villageois, de pouvoir porter à leur tour un uniforme les distinguant du reste de la population et leur conférant une autorité qu'ils disputent aux forestiers professionnels.

\section{Améliorer l'efficacité du service forestier}

La réforme de militarisation visait aussi « le renforcement de la discipline, de l'ordre et de l'efficacité dans l'exercice des tâches de police forestières, de police de chasse, de lutte contre le braconnage, de lutte contre les feux de brousse et de participation aux comités de défense ${ }^{18}$. Une rehausse substantielle du pouvoir d'achat des fonctionnaires, qui ont vu leurs salaires presque doublés grâce aux diverses indemnités liées à la perte de droits civils (droit de vote ${ }^{19}$, etc.), devait contribuer à les motiver davantage et à atteindre l'objectif recherché d'une plus grande rigueur dans la mise en œuvre de leurs activités. Cet objectif est resté un vœu pieux, car, selon les termes d'un ancien directeur des Eaux et Forêts, " un agent corrompu, être en uniforme ça le corrompt davantage ».

\section{La privatisation informelle des services forestiers}

Les ressources financières et matérielles destinées au fonctionnement du service forestier sur le terrain continuent à être gérées au niveau central, malgré des appels répétés à leur décentralisation venant des respon-

\footnotetext{
${ }^{18}$ Exposé des motifs de la Loi $n^{\circ} 2005-10$ du 3 Août 2005, République du Sénégal.

${ }^{19}$ Mais le Décret $n^{\circ} 2006-636$ du 14 juillet 2006 réintègre les militaires et paramilitaires dans le corps électoral.
} 
sables régionaux. Elles sont inégalement distribuées sur le territoire national : en règle générale, la direction privilégie les régions et les secteurs qui sont en position de produire des ressources financières importantes, et délaisse les régions "sèches ", qui présentent néanmoins des problèmes environnementaux aigus. En deuxième lieu, une grande partie de ces ressources reste au niveau central. Les services les plus démunis correspondent au niveau déconcentré du département (secteur), de l'arrondissement (brigade), de la communauté rurale (triages) et des postes de contrôle. Les agents de terrain n'ont souvent pas de moyens de locomotion, peu de carburant, pas de budget pour l'entretien et les réparations. Ils achètent leur propre uniforme aux autres corps militaires, ils fabriquent parfois eux-mêmes leurs propres galons. Les bureaux ne sont pas équipés ou peu fonctionnels, les postes de contrôle sont des abris de fortune. À titre d'exemple :

La brigade de M., dans la région de Tambacounda, ne dispose pas de bons véhicules. Le "double cabine " est en piteux état. Le parebrise est fissuré partout et tient par miracle, les clignotants manquent, la carrosserie est cabossée et le châssis déformé. Sur les trois motos du service, une seulement est fonctionnelle. Le chef de la brigade s'exclame: "Ici, on est des surghas, ce sont le IEF qui sont des patrons. Puisqu'ils restent tranquilles dans leurs bureaux, ils n'ont pas vu que les surghas sont les plus importants, car tout repose sur leur travail! On peut rester une année sans se voir » $(H$. S., 28/9/06). La brigade n'a pas de crédits de fonctionnement propres. Elle reçoit du secteur ou de la direction juste du carburant (35 l. d'essence et 80 l. de gasoil par mois) et de la fourniture de bureau. Il n'y a pas de téléphone, même pas receveur. Comme les agents ne veulent pas se déplacer constamment au télécentre du village, ils utilisent leurs portables personnels. La brigade n'a pas de locaux officiels, elle est installée dans un domicile privé ; le salon fait office de bureau de travail, de réception des usagers et aussi de garage pour les motos de l'équipe. L'électricité est payée par les agents, qui sont logés, à l'exception du chef de brigade, dans des chambres mises à disposition par le CERP. Le service n'est appuyé ni par la Communauté rurale ni par le programme d'aménagement forestier qui intervient dans la zone.

Dans ce contexte de pénurie structurelle, les services déconcentrés res- 
semblent à un archipel composé d'îlots fonctionnant en vase clos, avec peu de contacts formels avec les directions régionales et quasiment aucune relation avec la direction nationale : " la dernière fois que l'IREF est venu, il était en voyage et s'est arrêté juste pour pisser. C'est en cherchant l'interrupteur dans les latrines qu'il s'est rendu compte que je vis sans électricité ! ", s'exclamait le chef d'un sous-secteur dans la région de Kolda.

Les membres d'une équipe locale s'organisent pour s'équiper, individuellement et collectivement, afin d'assurer un fonctionnement minimal du service.

Individuellement, ils peuvent investir une partie des gains illicites obtenus grâce aux activités de police forestière pour s'acheter une moto, une nouvelle tenue ou de la fourniture de bureau. La corruption peut dans certain cas assurer la survie du service public. D'autres agents, comme le chef de brigade de R., s'adonnent à des activités génératrices de revenus pour financer les activités de la brigade :

"J'ai acheté une motopompe que j'ai placée au niveau du puits de la pépinière. Ceci me permet aujourd'hui de faire pousser des plants ornementaux et fruitiers dans la pépinière, et de pratiquer du maraîchage avec le personnel d'appui avec qui je partage les bénéfices tirés de la vente des légumes et des plants. Avec cet argent aussi, je paie mes factures d'eau et d'électricité qui ne sont pas prises en charge par le service. En cas de feux de brousse aussi, j'utilise cet argent pour me payer du carburant pour la moto pour me rendre aux lieux où le feu s'est déclenché. D'ailleurs, depuis que je suis là en début 2008, je n'ai reçu de la part du service que deux gros cahiers d'enregistrement des activités journalières comme fourniture au niveau de la brigade. À la place de ce que j'ai fait, mon prédécesseur avait préféré des poulaillers. " ${ }^{20}$

Collectivement, ils peuvent avoir recours à des mécènes locaux (opérateurs privés, entreprises privées, projets de développement) ou puiser

${ }^{20}$ Entretien mené par Coumba Ndoffène Diouf, le 28/9/2009. 
dans une " cagnotte ", une caisse commune alimentée avec une partie des ristournes légales issues des amendes et des recettes du contentieux.

Au-delà de l'origine des ressources financières mobilisées, cette situation amène les agents à considérer les équipements du service comme des biens privés : le chef de secteur de B., que j'ai rencontré dans un bureau fraîchement repeint et bien équipé, me disait avec fierté : " tout ce qui est ici, même les tampons des Eaux et Forêts, m'appartient. C'est moi qui l'ai acheté et je l'emporterai avec moi à mon départ ».

L'autonomie laissée de facto aux agents subalternes augmente leurs pouvoirs discrétionnaires déjà importants, et a des conséquences sur les logiques de redevabilité qui déterminent l'action des forestiers, comme nous l'avons noté précédemment. Couplée à la privatisation informelle des moyens logistiques des services, elle détermine un régime particulier d'activités sur le terrain.

Les brigades forestières, souvent composées d'un seul agent, partagent leurs activités entre le reboisement, la police forestière, la lutte contre les feux de brousse, la sensibilisation, la formation et l'encadrement des paysans. Dans les régions de Tambacounda et Kolda, les seules officiellement ouvertes à l'exploitation forestière, une part conséquente du temps de travail est en principe consacrée au suivi de ces activités. En revanche, les brigades situées dans les régions du vieux bassin arachidier sont davantage impliquées dans les opérations de mise en défens et de restauration des sols. La campagne de reboisement commence en avril et s'achève en septembre. La saison sèche (octobre - juin) est consacrée en revanche à la lutte contre les feux de brousse, le suivi de la campagne d'exploitation forestière et la police forestière. Le travail au bureau s'étale sur toute l'année, et comprend une permanence pour la délivrance des permis (circulation et dépôt) ainsi que la rédaction périodique de rapports d'activité.

On peut se demander si la militarisation du corps a renforcé sa dimension répressive. Une comparaison entre les journaux de bord de 1993 et de 2005 de la brigade de Koussanar, dans la région de Tambacounda, incite à une réponse nuancée. Si le pourcentage de journées consacrées aux activités de bureau reste stable (44,9\% en 1993 contre 45,3\% en 2005) et élevé 
dans l'absolu, il semble en revanche que les tournées de police forestière et de "ratissage" au niveau des forêts classées et des chantiers d'exploitation ont augmenté (de $8,8 \%$ des activités en 1993 à $11,6 \%$ en 2005). Par ailleurs, les agents en poste en 1993 étaient plus impliqués dans des tournées forestières sans but répressif (13,5\% des activités) que leurs collègues de 2005 (10,1\% des activités). Mais en 1993 la brigade de Koussanar a consacré 125 journées au contrôle routier et à la police urbaine ( $28,2 \%$ des activités), tandis que cette activité n'est plus déclarée comme telle en 2005. La différence est de taille en ce qui concerne le temps dédié au reboisement ( $2 \%$ en 1993 et $14,7 \%$ en 2005), à la lutte contre les feux de brousse par l'ouverture ou le nettoyage de pare-feux (rien en 1993, $9,4 \%$ des activités en 2005) et aux séminaires et réunions (0,9\% en 1993 contre 3,3\% en 2005). Dans ces domaines, l'équipe de 2005 semble beaucoup plus active que celle de 1993, même s'il faut préciser que le reboisement se réduit, en 2005, au travail à la pépinière de la brigade.

Des observations similaires faites auprès de la brigade de Missirah, toujours dans la région de Tambacounda, pour la période allant de janvier à septembre 2006, montrent que le travail de bureau reste toujours important ( $46,7 \%$ des activités), mais que le reboisement et le contrôle des chantiers sont ici privilégiés $(17,2 \%$ et $10,7 \%$ respectivement), au détriment des activités de répression (8,2\%). La présence de programmes d'aménagement forestier explique ces données, ainsi que le nombre élevé de réunions et séminaires (19 en 9 mois, soit 7,8\% des activités sur la période considérée) auxquels les agents ont pris part.

Si l'on descend au niveau d'un triage, celui de Wassadou (observations sur avril et septembre 2006), le seul agent en poste n'a pas consacré plus de $30 \%$ de son temps de travail aux activités de bureau, la police forestière (essentiellement des descentes dans le marché local, quelques visites inopinées dans la rôneraie et des " montages " sur la route nationale) constituant l'essentiel de ses tâches (42,5\%). Bien que ces données ne puissent être considérées comme représentatives de l'ensemble des brigades forestières au Sénégal, des observations de terrain que j'ai menées dans plusieurs régions du pays (Kaolack, Fatick, Diourbel, Saint-Louis) confirment que la militarisation n'a pas conduit à une augmentation notable des activités de police forestière. Cela ne traduit pas pour autant une conversion 
des agents forestiers au credo participatif. Cloués malgré eux à leur poste par manque de moyens ou de carburant, les agents tendent à n'aller sur le terrain que lorsqu'ils sont sûrs que la "chasse sera bonne ". Les sorties sont le plus souvent déterminées par les dénonciations d'un petit réseau d'informateurs locaux, ou alors se limitent à des "montages" inopinés dans des lieux de passage considérés comme stratégiques. Les opérations régulières de contrôle et de surveillance sont de facto très sporadiques, tant au niveau des espaces protégés que sur les chantiers de production de charbon de bois. En 2005, le chef du bureau du contentieux de K., chargé de suivre l'installation des surghas, avouait ne s'être jamais rendu sur le terrain depuis au moins deux ans. La même situation se vérifie au niveau des triages et des brigades: le chef d'un sous-secteur dans la région de Kolda, rencontré en 2005, disposait bien d'une moto, mais il refusait de l'utiliser pour des trajets supérieurs à 20 kilomètres " pour ne pas s'user ". Des observations conduites au marché de Diaobé ont également montré que le contrôle des déchargements des produits d'origine forestière était loin d'être systématique.

Dans ce contexte, la répression prime sur la prévention. Le jeune chef de secteur de B., rencontré en juillet 2009, me disait avoir constaté que ses agents préféraient se cacher et attendre que des " dégâts " soient commis pour ensuite sévir. "Mais en faisant cela ils ont perdu leur âme. Attraper la même personne tous les trois jours, c'est un constat d'échec, me disait-il. Notre action devrait empêcher les gens de récidiver ».

\section{Arrangements informels, simplification des procédures administratives et normes professionnelles pratiques}

Un corps paramilitaire pouvant exercer légitimement des activités de contrôle et de répression, doté de pouvoirs discrétionnaires importants (évaluation du délit et de la sanction à appliquer, large fourchette des amendes, etc.) sur des ressources économiquement et politiquement cruciales, soumis à des pressions contradictoires mais dépourvu des moyens nécessaires pour mener à bien son action, sont autant de facteurs, qui, réunis, constituent le socle idéal pour l'épanouissement de comportements corruptifs. 
Quelques travaux récents ont tenté l'inventaire des différentes formes de fraude ou de corruption qui gangrènent la gestion des ressources forestières au Sénégal (Fall et al., 2006, Djigo, 2006). Cependant, mes recherches m'incitent à essayer d'aller au-delà d'une lecture classique des dysfonctionnements en termes de néopatrimonialisme, de clientélisme et de "capture " des bureaucraties par les élites politiques et/ou économiques. Il ne s'agit pas de nier le constat d'un hiatus, parfois très important, entre les missions formelles de l'administration de l'environnement (le même constat étant par ailleurs valable pour la majorité des services administratifs du Sénégal) et leurs pratiques réelles. Le problème est que la majorité des travaux cités adoptent une approche à la fois normative et externaliste dans l'analyse des dysfonctionnements de l'administration. Elle est normative, dans la mesure où elle met l'accent sur les pratiques déviantes des agents de l'État par rapport aux normes formelles; elle est externaliste, puisqu'elle ne tient pas compte des logiques internes de fonctionnement de l'administration, mais elle s'appuie sur le point de vue d'acteurs externes, qu'il s'agisse des bailleurs de fonds ou des usagers de cette administration. J'ai en revanche essayé d'aborder cette question en adoptant le point de vue des agents des Eaux et Forêts qui ne sont pas avares d'exemples sur leurs propres pratiques et qui n'adoptent pas seulement, contre toute attente, une posture tendant à les justifier ou à en minimiser l'ampleur.

L'administration forestière gère des espaces et des ressources qui recèlent d'importants enjeux économiques : permis de chasse et amodiations, commerce de bois d'artisanat et de chauffe, collecte de fruits et d'essences sauvages, exploitation du charbon de bois, laquelle, à elle seule, "représente un chiffre d'affaires annuel de 20 milliards de FCFA pour l'État" (Boutinot, 2002). S'il a su bien gérer sa carrière, même un agent au bas de l'échelle hiérarchique peut arborer un niveau de vie incommensurable avec son salaire de base, tel cet ancien chef de secteur, transféré au poste de contrôle de Bargny en 2009 pour couronner un parcours qui lui a permis de financer un pèlerinage à la Mecque, bâtir une maison à Saint-Louis et une autre à Touba, et envoyer ses enfants en Europe pour leurs études supérieures. 
Les occasions d'enrichissement, particulièrement en zone d'exploitation, sont en effet nombreuses. Ceci n'est pas le lieu pour les décrire minutieusement. On pourra noter qu'elles se situent dans un continuum de pratiques aux frontières floues, qui vont du "péage " exigé dans chacun des quatorze postes de contrôle officiels ${ }^{21}$ que compte le Sénégal pour le visa gratuit du permis de circulation, à des " commissions ", en nature ou en espèces, résultant d'arrangements mutuellement convenus entre les agents forestiers et les producteurs pour contourner le code forestier. S'il est par définition impossible d'évaluer l'ampleur des transactions illicites, on ne se trompera pas en suggérant que les statistiques nationales en matière de contentieux ne recensent que l'infime partie des infractions réellement constatées mais dont le montant de l'amende est resté dans les poches d'un forestier sans qu'un reçu soit délivré : selon un haut cadre de la direction des Eaux et Forêts, on peut estimer à $80 \%$ le nombre de transactions réglées "à l'amiable ». Selon une étude récente, les $2 / 5$ des chargements de charbon qui parviennent à Dakar seraient d'origine frauduleuse (Djigo, 2006 :34). Les forestiers font aussi commerce des permis de circulation gratuits, qui sont en principe limités en nombre et réservés à un usage domestique. On sait également que, en cas de saisie de produits suite à un constat d'infraction, ces derniers sont revendus " aux enchères " à l'exploitant en infraction, au nom d'un autre membre d'une coopérative forestière. Les dépassements de tonnage sont aussi devenus la règle : les camions sont chargés généralement de 450 sacs au lieu des 300 réglementaires, ou l'on utilise des sacs pouvant contenir jusqu'à 80 kgs, au lieu de 50 kgs. De l'argent est distribué à chaque contrôle pour que les agents ferment les yeux.

Ces exemples sont loin d'épuiser la liste des pratiques frauduleuses qui font le lit des relations collusives entre agents forestiers, producteurs et usagers de l'administration. Plusieurs facteurs concourent à la corruptibilité des agents des Eaux et Forêts. En premier lieu, lorsqu'un forestier décide de verbaliser une infraction, il sait déjà qu'il risque d'être désavoué par un supérieur, à la suite d'une intervention politique, administrative ou

\footnotetext{
${ }^{21}$ Kahone, Pont Noirot, Gouloumbou, Koumpentoum, Mbar, Keur Ayib, Kaffrine, Sénoba, Pont Emile Badiane, Diourbel, Leybar, Khor, Fatick et Bargny. Le montant versé tourne autour de 2 000 FCFA par poste de contrôle, avec des variations toutefois selon la nature de la cargaison.
} 
religieuse, et que son action sera délégitimée aux yeux de la population.

\begin{abstract}
Au poste de contrôle de Bargny, au Sénégal, un camionneur déclarant transporter des sacs d'arachide avait dissimulé une cargaison de 450 planches de venn. Interpellé, il a exhibé un laissezpasser (permis de circulation) délivré à titre gratuit par l'IREF de Kolda. La cargaison frauduleuse appartenait à un général travaillant à la Primature. Dans un premier temps, I'IREF de Dakar a confirmé la confiscation du bois, mais les pressions politiques ont eu raison de l'agent et on l'a laissé partir (témoignage du chef de brigade de K., 4/10/06).
\end{abstract}

L'annulation d'une transaction réglementaire prive aussi l'agent verbalisateur et l'indicateur des ristournes prévues à cet effet par la loi ${ }^{22}$, et qui constituent une mesure incitative à agir de façon intègre. Mais même lorsque l'amende est normalement payée, l'agent forestier sait que les probabilités de toucher l'intégralité de ces ristournes sont très faibles. Au mieux, le service "fera cagnotte ": l'argent de cette caisse commune sera réparti entre tous les agents du secteur, histoire de motiver les agents qui " sortent ॥ rarement et de rétribuer le personnel auxiliaire, mais servira aussi à financer des frais de fonctionnement ou des dépenses à caractère social. Au pire, les ristournes finiront dans une caisse noire, gérée dans l'opacité la plus absolue par le chef du service et le responsable de la Caisse Intermédiaire des Recettes Forestières. Ainsi, "le fait de ne pas donner aux agents leurs ristournes a fait que nous préférons parfois régler les cas d'exploitation frauduleuse à notre niveau par arrangement " (ATEF chef de la Brigade forestière de R., le 28/09/09).

Le caractère relâché des relations avec les supérieurs hiérarchiques détermine aussi la teneur des actions sur le terrain : " la tentation est grande.

22 L'article R.64 de la loi $\mathrm{N}^{\circ}$ 98/03 du 8 janvier 1998 stipule que « Les trois dixièmes du produit des amendes, confiscations, restitutions, dommages-intérêts et contraintes sont attribués aux agents des Eaux et Forêts, aux agents commissionnés des Eaux et Forêts et, le cas échéant, aux agents des autres services habilités. La répartition est faite sur la base de deux dixièmes pour l'agent indicateur et du dixième pour l'agent verbalisateur. Les sept dixièmes sont versés à la collectivité locale gestionnaire de la forêt dans laquelle l'infraction a été relevée ou à l'Etat s'il s'agit d'une infraction dans le domaine forestier de l'Etat ». 
Personne ne me contrôle. Comment expliquer que depuis mon installation il y a six mois, personne, pas même l'IREF de K., soit venu me voir ? ", se demandait en juillet 2009 le chef de secteur de B., dans une région du bassin arachidier. "Il faut que l'agent sente la pression sur lui, sinon la distance et l'isolement l'amèneront à faire n'importe quoi ", ajoutait le chef de secteur d'un département limitrophe.

En outre, les agents s'abritent derrière la justification bien rodée selon laquelle le mauvais exemple vient d'en haut. Selon une rumeur qui parcourait le service en 2009, le directeur sortant des Eaux et Forêts aurait accordé à sa clientèle des autorisations spéciales de coupe allant jusqu'à 85000 quintaux, et aurait accordé à des " parents » des marchés publics pour la fourniture de gaines de très mauvaise qualité pour les pépinières : «Imaginez, un directeur qui fait ça! Comment se comporteront donc les agents d'un tel directeur ? " (chef de secteur de K., 27/7/2009).

C'est une évidence que les problèmes qui entachent la filière du charbon de bois et l'ensemble de la gestion des ressources forestières ont un caractère systémique, et que les agents subalternes ne sont que le dernier maillon d'une chaîne de fraudes et d'irrégularités qui remonte jusqu'à l'autorité de tutelle. En vérité, même les programmes financés par l'extérieur, et considérés comme des success stories, ne sont pas restés en dehors de ce système.

Selon un rapport d'inspection confidentiel portant sur le Programme de gestion durable et participative des énergies traditionnelles et de substitution (PROGEDE) de Tambacounda, le fonctionnement et les résultats du programme depuis son début auraient été passablement fourvoyés. Un système se serait créé sur place, avec la collusion des services forestiers, du personnel du PROGEDE (coordinateur et animateurs) et des présidents des CVGD et CIVGD. Beaucoup des CVGD inspectés n'étaient pas fonctionnels : les réunions n'étaient pas tenues, les comptes n'étaient pas à jour, leur gestion était monopolisée par le président. En l'absence de comptabilité et d'états de versement, les ristournes ${ }^{23}$ sur la vente du charbon de

${ }^{23}$ Pour leur clef de répartition, cf. Boutinot, dans ce même volume. 
bois auraient été massivement détournées. La collecte des fonds issus de la vente du charbon était faite par des individus non habilités, les agents forestiers ou le personnel du PROGEDE. On estime à 120 millions de CFA le montant annuel des ristournes qui se sont volatilisées.

La substitution du lieu d'exploitation était l'opération la plus courante : les produits forestiers venant de zones non aménagées étaient régularisés suite à la rédaction d'un constat de production fictif. Cette combine est hautement lucrative, car non seulement les exploitants vont travailler dans des zones où les coûts de production sont inférieurs, mais ils s'acquittent également d'une taxe forestière de 700 FCFA par quintal, au lieu des 1200 FCFA qu'ils devraient payer pour l'exploitation en zone non-aménagée. Le conseiller en aménagement, relevé de ses fonctions, aurait pour sa part créé un GIE d'exploitation forestière, basé à Bakel, et qui s'approvisionnait à Tambacounda. II est accusé d'avoir acheté du charbon sous couvert des comités intervillageois, d'avoir utilisé à des fins privées les moyens du service, et d'avoir détourné une partie des ristournes dues aux CVGD. Suite à ces enquêtes, la deuxième phase du PROGEDE a été bloquée, et l'on parle d'un transfert du programme sous la coupe du Programme national de développement local (PNDL).

En somme, la majeure partie de l'action administrative dans le secteur forestier emprunte la voie des arrangements informels avec les usagers. On pourrait même affirmer que l'administration forestière est un site important de production et reproduction de l'informalité, car l'action publique est menée par des acteurs qui naviguent dans une situation de pluralité des normes: des normes officielles (par exemple, le code forestier ou les statuts portant organisation interne du corps des Eaux et Forêts), des normes sociales (des principes généraux de conduite et de comportement dans la sphère sociale, plus ou moins codifiés), des normes professionnelles "pratiques", qui concernent des savoir-faire, des combines, des habitudes, des routines apprises "sur le tas " au cours de la socialisation professionnelle du fonctionnaire (par exemple, savoir dénicher une cargaison frauduleuse, savoir négocier le montant d'une amende, apprendre à traiter avec les autorités traditionnelles, etc.). 
Les différents dosages de ces normes contribuent à déterminer ce qu'on pourrait appeler la culture professionnelle des forestiers. Loin de tout déterminisme, cette culture professionnelle n'est ni figée, ni surplombant l'ensemble du corps. Elle n'est pas figée, puisqu'elle évolue au gré des changements de politiques publiques en matière d'environnement, des nouvelles approches du développement, et du renouvellement du personnel. En outre, plus qu'un ensemble de traits communs déterminant le comportement de tout agent de l'administration forestière, il est préférable de la penser tel un répertoire de normes, valeurs et registres d'action dans lequel peuvent puiser les forestiers dans leur pratique quotidienne par rapport à des enjeux traversant l'ensemble du corps administratifs (la réforme de décentralisation, la militarisation, les actions de développement, l'encadrement technique, etc.). C'est ainsi une partie intégrante de la culture professionnelle des forestiers que d'apprendre à traiter avec les différents types d'autorité, de marchander le montant d'une sanction légale, de respecter les codes de bienséance locaux, de déjouer et de prévenir les attaques en sorcellerie, de s'appuyer sur des intermédiaires (notables, indicateurs, autorités religieuses) dans leurs contacts avec la population.

\section{En guise de conclusion : réformes d'en haut et réformes d'en bas}

Redorer le blason du corps, retrouver la " grande famille forestière ", se protéger des pressions extérieures, renforcer l'ordre et la discipline, promouvoir la " bonne gouvernance ", mettre fin aux abus dans l'attribution des galons, rehausser les salaires des fonctionnaires : de tous ces objectifs, officieux ou officiels, seul le dernier semble avoir été couronné de succès, à tel point que la plupart des agents rencontrés parlaient volontiers d'une "militarisation alimentaire». Comme le regrettait en 2012 un des membres de la commission qui avait porté le projet de réforme du statut des Eaux et Forêts :

"Les agents étaient intéressés non pas par la discipline, mais par l'argent. Ils ont oublié tout le reste. Alors que les autorités auraient dû veiller à l'amélioration de l'efficacité du service, sinon c'est une perte pour l'Etat, qui donne plus d'argent pour plus d'efficacité. Et les directeurs qui se sont succédés n'ont pas veillé à cet aspect de la 
réforme. Une fois eue l'augmentation, la vie est belle et personne ne s'est soucié du reste. On a donc acquis de mauvaises habitudes, ça ne va pas être facile de les faire changer ».

Et pourtant, le service a besoin, outre une relève qui tarde à venir, d'une série de réformes qui lui permette d'assumer pleinement le rôle qui lui est dévolu dans la protection et la régénération des ressources naturelles. Pour l'heure, discrédité par rapport aux autres corps paramilitaires, menacé par la décentralisation, appauvri par la perte des projets et l'appui budgétaire, le service forestier a réagi sur plusieurs registres, mais qui semblent tous en contradiction avec les nouvelles missions qui lui ont été assignées: militarisation, renforcement de l'alliance avec les exploitants contre les élus locaux et les populations locales.

Mais l'on entrevoit, ici et là, l'émergence de micro-réformes par le bas, formalisant des habitus informels et qui promettent d'être plus efficaces. Le style de leadership du responsable d'un service (inspecteur régional, chef de secteur) joue ici un rôle prépondérant. Pour les forestiers, un bon chef doit savoir partager les moyens techniques et financiers avec ses subalternes; il est capable de "communiquer " et de les responsabiliser. II doit savoir défendre et protéger les intérêts des subordonnés et du corps en général, s'intéresser à leurs conditions de travail et écouter leurs exigences, savoir tenir tête au ministre, être peu politisé. En somme, un patron vis-à-vis des subalternes, mais sans pour autant être un client des supérieurs hiérarchiques.

On peut sans doute affirmer qu'un service caractérisé par des relations hiérarchiques de type patron-client est souvent mieux géré qu'un service où le chef entend appliquer à la lettre les critères de la " bonne gouvernance ". Les agents sont plus motivés, l'équipe plus soudée et plus à même de fournir un service de qualité. Ces relations internes aux services restent sur le plan de l'informalité. Toutefois, nous avons découvert que dans l'inspection de Tambacounda, le chef a pris l'initiative de formaliser l'emploi des ristournes, légales ou illicites, que les agents perçoivent lorsqu'ils verbalisent des infractions. Alors que traditionnellement ces gains étaient soit appropriés à titre individuel, soit versés dans une caisse noire et redistribués de façon clientéliste par le directeur régional, à Tambacounda les agents ont décidé de constituer une caisse, gérée par le comp- 
table, qui redistribue une partie des sommes selon une clé de répartition reconnue par tous et en utilise le restant pour financer des réparations urgentes, des besoins en carburant ou des dépenses imprévues à caractère social. C'est un exemple où la formalisation d'une pratique informelle semble améliorer le fonctionnement du service, prévenir les comportements de prédation et souder la solidarité interne. Le système a été repris à Saint-Louis, où l'inspecteur a été muté récemment. Mais à Tambacounda, à son départ, il a été abandonné, signe que la volonté du chef est prépondérante dans la mise en œuvre d'une micro-réforme locale.

Dans d'autres régions situées en dehors de la zone charbonnière, nous avons rencontré des jeunes chefs de secteur qui, tout en ayant complètement privatisé leur service, rejettent en même temps la culture professionnelle classique des Eaux et Forêts, surtout dans sa forme du patronage interne, de la corruption et de la répression des usagers. Ils ont ainsi entrepris des petites réformes visant à formaliser, pour mieux les contrôler, les arrangements informels que leurs prédécesseurs avaient instaurés avec la population. $\mathrm{Si}$, comme aiment à le rappeler les forestiers, " le charbon est noir et l'on se salit les mains", alors le moteur de transformation interne du corps des Eaux et Forêts pourrait venir de ces mêmes "zones sèches » pendant longtemps déconsidérées par le service.

\section{Bibliographie}

AIGREF (Association des ingénieurs du Génie rural, des eaux et forêts), 2001, Des Officiers royaux aux ingénieurs d'État dans la France rurale (1219-1965). Histoire des corps des Eaux et Forêts, Haras, Génie rural, Services agricoles, Paris, éditions Tec et Doc, $690 \mathrm{p}$.

BELLOUARD, P., 1955. "La situation forestière de l'Afrique Occidentale Française ", Bois et Forêts des Tropiques, no 39, janvier-février, pp. 9-23.

BIERSCHENK, T., 2010, "States at Work in West Africa : Sedimentation, Fragmentation and Normative Double-Binds ", Working Papers of the 
Department of Anthropology and African Studies of the Johannes Gutenberg University, Mainz, 113.

BLUNDO, G., 2011a, «Une administration à deux vitesses. Projets de développement et construction de l'Etat au Sahel ", Cahiers d'Etudes Africaines, LI (2-3), 202-203, pp. 427-452.

BLUNDO, G., 2011b, «Comme un ballon de foot. La gestion quotidienne des ressources humaines dans les services forestiers en Afrique de l'Ouest ", in Nikolaus Schareika, Eva Spies et Pierre-Yves LeMeur (eds.), Auf dem Boden der Tatsachen. Festschrift für Thomas Bierschenk, Köln: Köppe Verlag (Mainzer Beiträge zur Afrikaforschung 28), pp. 377-394.

BLUNDO, G., 2012, « Le roi n'est pas un parent. Les multiples redevabilités au sein de l'État postcolonial en Afrique ", in Pascale Haag, Cyril Lemieux (dir.), Faire des sciences sociales, vol. 1, Critiquer, Paris, Éd. de I'EHESS (coll. « Cas de figure »), p. 59-86.

BLUNDO, G. \& GLASMAN, J., 2013, "Introduction: Bureaucrats in Uniform », special issue of Sociologus. Journal of Empirical Social Anthropology, no 63, pp. 1-9.

BLUNDO, G. et J.-P. OLIVIER DE SARDAN, 2007, " La corruption quotidienne en Afrique de l'Ouest ", in Blundo, G. et J.-P. Olivier de Sardan (sous la dir. de), Etat et corruption en Afrique. Paris, Karthala, pp. 79-117.

BOUTINOT, L., 2002, "La gestion décentralisée des ressources forestières au Sénégal : transfert de compétences et transfert de pouvoir $"$, Bulletin de I'APAD, 22, pp. 27-45.

BOUTINOT, L., 2005, "La décentralisation de la gestion des ressources forestières au Sénégal : un processus contraint par le marché ? ", Bulletin de l'APAD, 26, pp., 47-66.

BOUTINOT, L. \& DIOUF.C.N., 2006, «Quand certaines approches participatives engendrent des formes ambiguës de mobilisation de la société civile ", in Bertrand, A., Montagne, P. et Karsenty, A., L'Etat et les politiques forestières en Afrique francophone, Paris, L'Harmattan, pp 195-212.

BOUTINOT, L. \& DIOUF, C.N., 2007, « Les linéaments de la politique forestières dans les normes de régulation institutionnelle de la filière du bois énergie au Sénégal ", Afrique Contemporaine, 222- 2, pp. 45-72.

BUTTOUD, G. ,1995, La forêt et l'État en Afrique sèche et à Madagascar. Changer de politiques forestières, Paris, Karthala. 
CANTENS, T., 2009, " Être chef dans les douanes camerounaises, entre idéal type, titular chief et big katika ", Afrique Contemporaine, 2 (230), pp. 83-100.

DJIGO, A S., 2006, Etude sur la surveillance, le suivi, et le contrôle de l'exploitation forestiere au Sénégal. Programme agriculture/gestion des ressources naturelles 'Wula Nafaa', septembre.

FAIRHEAD, J. \& LEACH, M., 2000, « Desiccation and Domination: Science and Struggles over Environment and Development in Colonial Guinea », The Journal of African History, Vol. 41, No. 1, pp. 35-54.

FALL, A. S., ed. 2006, Gouvernance et corruption dans le domaine des ressources naturelles et de l'environnement au Sénégal. Dakar, Forum Civil.

FOURY, P., 1953. "Politique forestière au Sénégal ", Bois et Forêts des Tropiques, no 30, juillet-août, pp. 8-21.

GLASMAN, J., 2011, Les corps habillés. Genèse des métiers de police au Togo, thèse en histoire, Université Paris 7 et Université de Leipzig, 549 p.

KANTE A.M., 2006, Décentralisation sans représentation. le charbon de bois entre les collectivités locales et l'Etat. Dakar, Codesria, Série de Monographies.

KANTE A.M., 2009, Décentralisation, gouvernance forestière et démocratie au Sénégal : y a-t-il un avant et un après 1996 ? In : VertigO : La revue en sciences de l'environnement, hors série 6, ACFAS, mai 2009.

NADER, L. 1974. Up the anthropologist - perspectives from studying up. In Hymes, Dell, ed. Reinventing Anthropology. New York, Vintage, 284-311.

RIBOT, J.C., 2001. "Science, Use Rights and Exclusion: A History of Forestry in Francophone West Africa », Drylands Programme Issue Paper No. 104, International Institute for Environment and Development (IIED).

RIBOT, J.C., 2008, «La non-décentralisation démocratique au Sénégal: le nontransfert de l'autorité sur les forêts ". Séries de documents de travail sur la représentativité, l'équité et l'environnement 23. Washington, WRI.

RIBOT, J. C., 2009, « Authority over Forests: Empowerment and Subordination in Senegal's Democratic Decentralization ", Development and Change, 40: 105-129.

SEGALINI, C., 2012, "Les agents des Parcs Nationaux au Sénégal: soldats de la participation? ", Politique Africaine, no 128, 101-119. 
WEDEL, J., SHORE, C., FELDMAN, G. and S. LATHROP, 2005, "Toward an Anthropology of public policy ". The Annals of the American Academy of Political and Social Science 600: 30-51.

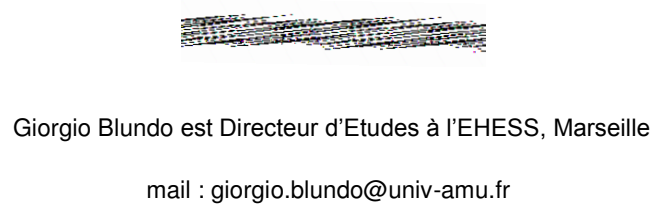

mail : giorgio.blundo@univ-amu.fr 